\title{
Services for younger sufferers with dementia
}

\section{K. C. M. Wilson and José Ferran}

\section{Prevalence}

Prevalence and incidence rates of dementia in people under the age of 65 are difficult to determine. Surveys differ in design, examine different population samples and include varying levels of disease in estimating rates. Most of the surveys are small and are conducted through case note review.

In 1982 Whalley estimated a prevalence of $0.1 \%$ of Alzheimer's disease in the population under the age of 65 . This has subsequently been adopted by the Alzheimer's Disease Society in its drive to draw attention to the sufferers of the syndrome (Alzheimer's Disease Society, 1991a, 1993).

Two meta-analyses have included data on the prevalence of dementia in populations under the age of 65. Jorm et al (1988) estimated prevalence rates of dementia to be $0.7 \%$ in the $60-64$ age group. More recently, the EURODEM prevalence research group (Rocca et al, 1991) found prevalence of Alzheimer's disease to be $0.02 \%$ for the age group of 30-59 and $0.3 \%$ for the $60-69$ group.

By examining medical notes across Scotland, McGonigal et al (1993) found an incidence of probable Alzheimer's disease of 22.6 per 100000 and 40.5 per 100000 for broad Alzheimers disease in 40-64-year-olds. Women had a greater risk of developing the syndrome. Densely populated areas had a higher prevalence than rural areas and vascular dementia was associated with lower socioeconomic class. Both types of dementia were associated with paternal occupation of coal mining.

Newens et al (1993) conducted a retrospective study of both psychiatric and general hospital case notes and other documentary sources in the Northern Health Region. The estimated point prevalence of presenile Alzheimer's disease (4564) was 34.6 per 100000 with an annual incidence of 7.2 per 100000 . A similar prevalence of early onset Alzheimer's and multi-infarct dementia was found by Delany \& Rosenvinge (1995) in Southampton. This study identified cases through accessing statutory and voluntary agencies and an advertisement was also placed in the local paper.

The variation between studies in incidence and prevalence rates may be a consequence of sociodemographic and geographical variations (Whalley et al, 1995). However, methodological differences between studies almost certainly contribute. Most of the studies identified cases through examination of case notes and are retrospective in design. Many restrict case identification to Alzheimer's disease and vascular dementias and exclude other diseases presenting as dementia in this age group. Studies employ different age groupings and diagnostic categories as well as varying in the stringency of defining criteria for each disorder.

\section{Psychosocial problems and carer stress}

Despite most of the surveys being limited to Alzheimer's disease and vascular dementias, a wide variety of disorders may present as dementia syndrome before the age of 65 . These include alcohol-related disorders, frontal lobe dementias, and affective disorders presenting as 'pseudodementias'. Such disorders are associated with considerable psychiatric and behavioural disturbance, inflicting considerable financial and social distress on families and carers.

K. C. M. Wilson is Senior Lecturer in Psychiatry of Old Age, with sessional responsibility for development of services for younger sufferers of dementia in the Liverpool area, and affiliated with EMI Academic Unit, St Catherine's Hospital, Church Road, Birkenhead, Wirral L42 0LQ. José Ferran, MRCPsych, is a Lecturer in Psychiatry of Old Age at the University of Liverpool. He is currently conducting a longitudinal study of psychiatric phenomena and service need in younger sufferers of dementia. 
A number of studies have found high levels of behavioural disturbance and dependency (Baldwin, 1994; Sperlinger \& Furst, 1994; Delany \& Rosenvinge, 1995; Newens et al, 1995). In Baldwin's sample $(n=43)$ half the cases received neuroleptics or antidepressants. Nearly half of the 109 cases of Alzheimer's disease in Newen's study were dependant in all six areas of activities of daily living that were examined. In those studies (Baldwin, 1994; Sperlinger \& Furst, 1994; Delany \& Rosenvinge, 1995) that recorded employment status, nearly all sufferers were unemployed and three-quarters of Sperlinger \& Furst's sample had to retire prematurely because of their illness (total sample 15 cases).

All studies draw attention to the burden of caring for sufferers of dementia in younger people. Over half the carers of sufferers living in the community in Baldwin's study were rated as 'cases' by the General Health Questionnaire (Baldwin, 1994). Likewise, Sperlinger \& Furst (1994) draw attention to the high level of stress experienced by the carers and the need for experienced counselling. Half the carers in this study and nine of 27 in Delany \& Rosenvinge's sample had to change job or retire through caring for the sufferer, imposing considerable financial burden on the family unit.

\section{Surveys of service provision}

In many areas of the country, dementia services cater for people over the age of 65 , specifically excluding younger sufferers. In other areas, younger sufferers are forced into these services, irrespective of service suitability and individual needs of the patient and carers. In other situations diagnostic services are provided and little followup is arranged. The numerous anecdotal reports that describe the difficulty that carers and patients have in accessing services highlight the loneliness, sense of isolation and lack of support (Cox \& Mclennan, 1994; Mulligan, 1994).

In 1993 the Alzheimer's Disease Society found that only $2 \%$ of sufferers in this age group had access to specialised services, including day care, respite and home care. Towards the end of 1994 (Alzheimer's Disease Society, 1994), the Society conducted a postal survey of its local branches concerning the provision of services for younger sufferers in England, Wales and Northern Ireland (see Box 1). Responses were received from 68 branches. Seventy-two per cent of the branches reported that their local health authority had not identified a consultant with responsibilities for diagnoses, assessment and management of youn-
Box 1. Summary of Alzheimer's Disease Society survey

There are about $\mathbf{1 0 0 - 1 5 0 ~ s u f f e r e r s ~ i n ~ e a c h ~}$ district health authority

Younger sufferers receive services designed for older patients

Access to services is patchy

Health authorities lack knowledge of prevalence and service need

Few authorities have clear policies for referral and care

Less than one-quarter of branches were aware of genetic counselling facilities

Specialist services not identifed in $87 \%$ of branches

ger people with dementia. This lack of organisation is reflected in the finding that only $22 \%$ of branches were able to identify genetic counselling services for the families suffering from familial dementias. Nearly all the branches reported that Health Authorities had yet to carry out an investigation into the service needs of younger people with dementia. These findings are obviously reflected in the relative lack of day care facilities, domiciliary services, respite and appropriate training for professionals working with this patient group. In those authorities that had conducted such surveys, local, specialised services were more likely to have been developed.

The lack of coordination and service planning highlighted in the Alzheimer's Disease Society report may contribute to the delay in referral to secondary services and the lack of appropriate counselling experienced by most sufferers (Spurlinger \& Furst, 1994). Even when patients are referred to secondary services, Baldwin (1994) found that many sufferers received inadequate services and did not have access to long-term psychiatric and social follow-up. Despite most patients in Delany \& Rosenvinge's study ( 23 out of 27 cases) having been referred, the authors found considerable unmet need with particular reference to the lack of counselling and social support. Newens et al's (1995) larger survey draws attention to the same issue. Only $39 \%$ of carers had met a community mental health nurse, few cases had support from district nurses for management of incontinence and over half the sample saw their general practitioner less than once a year.

In most of these studies, the majority of patients were referred to services for the elderly mentally ill. This can be distressing for younger patients, and patients with Huntington's Chorea in particular 
(Baldwin, 1994). In Delany \& Rosenvinge's (1995) study, four out of 11 cases were distressed by attendance at a day centre for older people (the mean age of the sample was 54). Twenty-one per cent of Newen's (1995) sample attending day centres had similar experiences.

Services designed for older dementia sufferers rarely have the resources to provide formal neuropsychological assessment, sophisticated functional neuro-radiological scanning or have ready access to genetic counselling and child support services, which may be indicated in the diagnoses and management of some cases. Attention is drawn to these issues by Allen \& Baldwin (1995). The authors compared the diagnostic performance of elderly mental illness services with those of a specialist neurological service in the same region. The elderly mental illness services were less likely to offer appropriate neuro-radiological or formal neuropsychological testing in establishing the diagnoses. They conclude by advocating a multi-professional service in which neurologists and psychiatrists complement each other in diagnoses and long-term follow-up.

The lack of services, isolation of carers and difficulty in accessing what services exist has led to carers promoting a Declaration of Rights for Younger People with Dementia and their Carers (Alzheimer's Disease Society, 1991b). This document makes a number of reasonable demands. These include: access to welfare benefits (and retrospective reinstatement of rights and benefits for those that have lost their employment); a fully informed medical assessment, and implementation of care planning and management. Local services conducive to the needs of this age group are demanded, with particular reference to day care and respite. Lastly, appropriate awareness and training is advocated for public and professionals alike. The document has been adopted by the Alzheimer's Disease Society and has been a cornerstone in its campaign for service development.

\section{Merseyside surveys}

Two adjacent authorities have conducted surveys of need in the Merseyside area (Wilson \& Johnson, 1991; Wilson et al, 1994). Both surveys included all cases under the age of 65 diagnosed as suffering from dementia syndrome and excluded patients with learning disability, sufferers from head injuries and HIV-related dementias. These surveys have led to service development and subsequent audits of service delivery.

\section{Survey of medical and service need (Liverpool)}

The initial developments in Liverpool were underpinned by a longitudinal survey of patients identified within the social and health services by encouraging referral to a psychogeriatrician with a mandate to develop services (Wilson \& Johnson, 1991). Fifty-nine patients were identified. Referring agents included general practitioners, community mental health nurses, general psychiatrists, hospital liaison services, research organisations, selfreferrals and social services. Less than half of the cases had appropriate medical or psychiatric diagnostic assessments. Service delivery to 39 cases was recorded over six months. All but one of the subsample lived at home. Eleven cases were in contact with secondary medical services. Eight cases had social services contact. The remainder had no planned follow-up, counselling or help.

All cases required access to at least one of the community services available to dementia sufferers over the age of 65 in Liverpool. The greatest demand was for community mental health nurse services, social work intervention, appropriate day care facilities, domiciliary care attendants and respite services. This high level of demand reflected high levels of functional and behavioural morbidity. Twenty-one cases suffered from profound levels of anxiety and associated 'clinging' behaviour with incessant following of their carers. Seven cases had alcohol problems, five cases suffered from concomitant depressive illness and three cases suffered from hallucinations and delusions.

Ten cases were admitted into institutional care over the six month follow-up period. Two cases were admitted into old age residential homes for respite. Six cases required admission into psychiatric services for acute treatment (one under the Mental Health Act).

\section{Survey of prevalence and social needs (Sefton survey)}

The local authority purchasers commissioned a survey of the social needs of sufferers and their carers in the adjacent borough to Liverpool. The aim of the survey was to estimate the point prevalence of younger sufferers with dementia in the borough and to describe the social needs of the cohort. The Borough of Sefton has an approximate population of 88500 between the ages of 40 and 64 .

The prevalence of dementia syndrome in patients under the age of 65 was established through networking voluntary, social and health agencies, identifying individuals through the regional memory clinic and interviewing managers of private, voluntary and social service institutions. One hundred and one cases were identified. Fiftythree cases lived in long-term care institutions (notably, many managers and care staff did not know the meaning of dementia, and were likely to 
exclude patients not specifically labelled as Alzheimer's disease sufferers). Nineteen cases residing in private institutions had been imported from addresses outside the borough.

Twenty-six patients and carers were interviewed. Sixteen sufferers lost employment through the dementia. All but six cases lacked personal pensions and few carers were able to maintain employment. Visits from friends and neighbours were rare and the carers felt isolated. The requirement for services were similar to those found in the Liverpool survey with emphasis on day care and personal, domiciliary care.

\section{Service development on Merseyside}

Until 1989 services for patients suffering from dementia under the age of 65 in Merseyside fell between Old Age Psychiatry services, General Adult Psychiatric services and a wide variety of other services. There was no focus for referral, no one service claimed responsibility and service providers failed to communicate with one another. The Alzheimer's Disease Society initiated a branch meeting, with the support of health and social services to examine the issues. From this meeting the Declaration of Rights was generated.

At the same time the Special Mental Illness Grant provided funding to launch a community facility (Croften Mews Project) run by Liverpool Social Services and an associated community mental health nurse. The community facility provides a specialised day centre, has its own transport, and services patients and their families at home through domiciliary care support workers. At any one time the centre services up to 21 patients. A consultant in Old Age Psychiatry with sessional commitment for early onset dementia services was appointed. A fulltime social worker has recently been appointed to manage early onset dementia in the District of Liverpool.

Subsequent developments include the establishment of a cognitive function clinic led by a consultant neurologist, supported by the appointed consultant in old age psychiatry, a psychologist and appropriate diagnostic facilities provided by the Regional Centre for Neurology and Neurosurgery. The adjacent local authority (Sefton) has developed services designed to cater for the needs of the local population. A domiciliary resource centre, providing domiciliary aids, specialising in this client group has been developed. Subsequent developments include the establishment of specialised day centre facilities and the appointment of a full-time social worker.

Throughout development of the services, health and social services, the university, voluntary agencies and patient groups have collaborated in strategic
Box 2. Services in Merseyside

Regional service: diagnostic

Neurologist

Psychologist

Psychiatrist

Regional service: counsellors

Geneticist

Child counsellors

District services $(\times 2)$

Community mental health nurse

Social worker

Domiciliary worker

Day care facilities

Session consultant psychiatrist

planning, audit, service delivery, counselling and education. A Merseyside-wide perspective has been taken with a view to identifying needs in different authorities and establishing individuals (health and social services) with responsibility to build expertise, identify services that are available and develop services where indicated (Box 2).

Both local and other surveys have indicated that development should prioritise community services. The Early Onset Dementia services establish a diagnosis and provide community follow-up. Arrangements between consultants in General Adult Psychiatry, Old Age Psychiatry, the Brain Injury services and Community and Rehabilitation services provide in-patient facilities when necessary. Long-term care facilities are provided by private-sector nursing and residential homes. Placing younger, active sufferers in nursing homes can be problematical. Over time, a number of nursing homes have developed skills in management of this client group and are supported by the community team.

\section{Audit of patients attending the Merseyside Cognitive Function Clinic and follow-up services}

\section{The clinic}

The clinic was set up in 1992 as a diagnostic service for patients presenting with cognitive dysfunction under the age of 65 . An advisory service is provided for patients with brain injury, learning disability and HIV-related dementias as comprehensive services already exist for these patient groups. 
The Cognitive Function Clinic is based at the Merseyside Centre for Neurology and Neurosurgery. The clinic has access to both in-patient and out-patient facilities, including CAT and NMR scanning.

Patients are asked to attend for a day, during which they receive a neurological examination and physical investigations, followed by psychometric testing. Most patients receive a CAT scan and are then interviewed by a psychiatrist in the afternoon. Cases are reviewed by all parties at the end of the clinic. Diagnosis is allocated to ICD-10 criteria (World Health Organization, 1992). Psychiatric follow-up is provided by the Consultant Psychiatrist in Old Age Psychiatry and a community mental health nurse, with the support of the local authority specialist social services. Those patients living outside the Liverpool and south Sefton areas are referred to the appropriate psychiatric services.

\section{Diagnostic review}

A retrospective review of the diagnoses of a consecutive series of 200 cases was undertaken. The diagnoses established after an average of one year follow-up were compared with the diagnosis with which the patient was initially referred and the diagnosis allocated to the patient after first assessment at the cognitive function clinic. Just under one-third of cases presenting to the clinic were found to have diagnoses of Alzheimer's disease. This condition was accurately diagnosed by the referring agents. Seventeen per cent were diagnosed as suffering from vascular dementia. This condition was under-diagnosed by the referring agents. A similar percentage was found to be suffering from depression, presenting with cognitive change. This was uniformly underdiagnosed by the referring agent. Alcohol dementias presented as the next largest diagnostic group. Overall, $53 \%$ of patients were referred with the wrong diagnoses or with no diagnosis having been made. Notably, $22.8 \%$ of cases needed a follow-up interview some months later in order to confirm a final diagnosis.

\section{Psychiatric and behavioural symptoms}

Ready access to documentation, medical and nursing notes enabled fairly detailed study over follow-up of an average of one year. Nearly half of the cohort suffered from major depressive disorder during the follow-up period. Aggression (27\%), insomnia $(30 \%)$, anxiety (29\%) and wandering (25\%) were common experiences. Poor mobility, incontinence and agitated behaviour were less common findings. Patients suffering from alcohol dementia were particularly prone to fire hazard (55\%).

\section{Service use review}

The relatively high prevalence of psychiatric and behavioural disorders resulted in frequent use of health and social services. Use was recorded in 138 cases living in the Liverpool area and receiving follow-up by the specialised psychiatric and social services. Sixty per cent had been referred to the community mental health nurse and $40 \%$ had been referred to the social worker. In total, $22 \%$ of cases used the day centre facilities and another $16 \%$ utilised domestic services. A surprisingly large proportion (24\%) were admitted to acute medical and surgical wards for treatment during the year of follow-up. Thirteen per cent were admitted to acute psychiatric and psychogeriatric services for treatment (three under the Mental Health Act). Twenty-two per cent were admitted to long-term care facilities.

\section{Observations}

The diagnostic review of cases presenting to the clinic illustrates the difficulty in establishing a diagnosis in many of the cases presenting. Notably, $18 \%$ of patients were found to be suffering from depressive disorders, presenting with cognitive impairment. In following-up the cohort, it is apparent that younger sufferers with dementia experience a high level of functional and behavioural disturbance. Individualised packages of care draw on services provided by acute adult psychiatric services, psychiatry of old age services and both 'specialised' and non-specialised social services. The community mental health nurse and social worker played an integral part in facilitating care plans across services, drawing on an increasing experience and knowledge base. Both community agents played important advisory roles, liaison with other agents, facilitation of discharge plans from in-patient units and arrangement of the most appropriate long-term care in institutions.

\section{Conclusions}

At a national level, the response to inadequate service provision has been led by the Alzheimer's Disease Society. The Society has established a steering committee to examine the issues and has appointed a full-time officer to facilitate the response. The Society is working closely with service developers across the country. The National Hospital for Neurology and Neurosurgery (Queens Square) is collaborating with the Society in establishing a general advice and information service with a national remit for carers and sufferers of early onset 
dementia (Counselling and Diagnoses in Dementia: CANDID).

At the local level, a number of surveys have been conducted. Prevalence figures vary between studies as a consequence of differences in study design, the illnesses examined and the criteria by which each illness is identified. At least one study suggests that prevalence rates of Alzheimer's and vascular dementias may be influenced by socioeconomic variables. Our experiences in Liverpool suggest that surveys restricted to the identification of Alzheimer's and vascular dementias in this age group will identify approximately half of all dementia sufferers. Other diagnostic groups including alcohol dementias, Huntington's and frontal lobe dementias experience considerable behavioural and psychiatric problems with little support and care.

Health and social services will vary in profile and the degree in which they cater for the needs of this relatively small group of sufferers and their families. The surveys indicate that diagnosis is difficult and patients and their families require comprehensive follow-up. What evidence there is suggests that few providing or purchasing units have considered the issue. Those surveys that have been conducted suggest that varying proportions of sufferers and their families are falling between services and are not receiving appropriate care.

The management of individuals and their families involves close liaison between services and professions. In many cases psychiatrists specialising in old age psychiatry accept this responsibility even though they are not funded to do so. Links with neurologists, genetic counsellors, child and family counsellors, and community services catering for younger patients and their families tend to be poorly organised with little or no overall coordination or planning.

We have found that these organisational issues are best managed through the establishment of a social worker and consultant, supported by a community mental health nurse as a point of referral for sufferers within a defined population. This small team coordinates the care plans and services for younger sufferers in Liverpool. As experience grows, the strengths and weaknesses of the local services can be assessed and deficits addressed. In Liverpool and Sefton local authorities day care facilities and outreach carers have been developed. We have found that one session per week of a consultant's time, supported by a fulltime community mental health nurse and social worker provides the nucleus of the community team, supporting a population of approximately 440000 .

\section{References}

Allen, H. \& Baldwin, B. (1995) The referral, investigation and diagnoses of presenile dementia: Two services compared. International Journal of Geriatric Psychiatry, 10, 185-190.

Alzheimer's Disease Society (1991a). Younger Onset Dementia. Information Pamphlet. London: ADS.

- (1991b) Declaration of Rights for Younger People with Dementia and Their Carers. London: ADS.

- (1993) Deprivation and Dementia. Report. London:ADS.

- (1994) Services for Younger People with Dementia. Report. London: ADS.

Baldwin, R. C. (1994) Acquired cognitive impairment in the presenium. Psychiatric Bulletin, 18, 463-465.

Cox, S. \& Mclennan, J. (1994) A Guide to Early Onset Dementia. University of Stirling: Dementia Services Development Centre.

Delany, N. \& Rosenvinge, H. (1995) Presenile dementia: sufferers, carers and services. International Journal of Geriatric Psychiatry, 10, 597-601.

Jorm, A. F. \& Korten, A. E. (1988) A method of calculating increases in the number of dementia sufferers. Australian and New Zealand Journal of Psychiatry, 22, 183-189.

McGonigal, G., Thomas, B., McQuade, C., et al (1993) Epidemiology of Alzheimer's presenile dementia in Scotland, 1974-1988. British Medical Journal, 306, 680-683.

Mulligan, E. (1994) Out of place and out of time. Journal of Dementia Care, 2, 23-25.

Newens, A. J., Forster, D., Kay, D., et al (1993) Clinically diagnosed presenile dementia of the Alzheimer's type in the Northern Health Region: Ascertainment, prevalence, incidence and survival. Psychological Medicine, 23, 631-644.

-, Forster, D. P. \& Kay, D. (1995) Dependency and community care in presenile Alzheimer's disease. British Journal of Psychiatry, 166, 777-782.

Rocca, W., Hofman, A., Brayne, C. et al (1991) Frequency and distribution of Alzheimer's disease in Europe: A collaborative study of 1980-1990, prevalence findings. Annals of Neurology, 30, 381-390.

Sperlinger, D. \& Furst, M. (1994) The service experiences of people with presenile dementia: A study of carers in one London Borough. International Journal of Geriatric Psychiatry, 9, 47-50.

Wilson, K. \& Johnson, F. (1991) Early Onset Dementia Service: A Case of Need in Liverpool. Birkenhead: St Catherine's Hospital.

Wilson, K., Ferran, J., Johnson, F., et al (1994) A Survey of Prevalence and Social Need of Younger Sufferers with Dementia. Birkenhead: St Catherine's Hospital.

Whalley, L. J., Carothers, A. D., Collyer, S., et al (1982) A study of familial factors in Alzheimers Disease. British Journal of Psychiatry, 140, 249-256.

-, Thomas, B. M. \& Starr, J. M. (1995) Epidemiology of presenile Alzheimer's disease in Scotland (1974-1988). Exposures to possible risk factors. British Journal of Psychiatry, 167, 732-734.

World Health Organization (1992) The Tenth Revision of the International Classification of Diseases and Related Health Problems (ICD-10). Geneva: WHO.

\section{Multiple choice questions}

1. Epidemiological studies of Alzheimer's disease in under-65s demonstrate that:

a there is a prevalence of $40.5 / 100000$ for 'broad' Alzheimer's in Scotland

b women are at greater risk of developing the syndrome 
c an incidence of 7.2/100 000 has been found in the Northern Health Region

$\mathrm{d}$ the prevalence decreases with age in people under the age of 70

e Scottish studies show that the prevalence is independent of population concentration.

2. Surveys of psychosocial morbidity demonstrate that:

a $25 \%$ of cases of Alzheimer's disease in the Northern Health Region were dependant in at least 6 areas of activities of daily living

b at least one study has shown that half of younger sufferers are receiving neuroleptic medication

c the GHQ demonstrates that a minority of carers are suffering to the extent that they are identified as 'cases'

d sufferers from alcohol related dementias are particularly prone to fire hazard

e aggression is found in up to $10 \%$ of sufferers of early onset dementia.

3. Surveys of services for younger sufferers demonstrate that:

a half of local health authorities had not identified a consultant with responsibility for services for younger sufferers of dementia

b services tend to be fairly well coordinated across the country

c $10 \%$ of sufferers and their carers are discontented with day services catering for older people

d the majority of cases receiving non-specialised services receive appropriate counselling and follow-up

e up to $60 \%$ of cases in the Liverpool longitudinal study were referred to the community mental health nurse.

4. In diagnosing the illness:

a younger sufferers referred to old age psychiatry services are less likely to be investigated with neuro-radiological and formal psychological testing than patients referred to a specialist diagnostic centre

b sufferers known to non-specialised social and health services have received appropriate diagnostic services

c Alzheimer's disease and vascular dementias account for $75 \%$ of the diagnoses of younger sufferers presenting to a regional diagnostic service

d referring agents establish the correct diagnosis in $75 \%$ of cases referred to the diagnostic services

e $10 \%$ of cases require more than one diagnostic assessment before a diagnosis can be confirmed.

5. Service developments:

a CANDID is a local organisation servicing patients attending Queens Square, London

b the Alzheimer's Disease Society has a liaison officer, specifically designated for younger sufferers with dementia

c most purchaser and providing units have considered the needs of younger sufferers in their business planning cycle

d surveys demonstrate that sufferers have ready access to genetic counselling services

e service development should concentrate on establishing in-patient services with later development of community services.

\begin{tabular}{|c|c|c|c|c|}
\hline \multicolumn{5}{|c|}{ MCQ answers } \\
\hline 1 & 2 & 3 & 4 & 5 \\
\hline a $T$ & a F & a $F$ & a $T$ & a $F$ \\
\hline b $T$ & b $\mathrm{T}$ & b F & b F & b $\mathrm{T}$ \\
\hline c $\mathrm{T}$ & c $F$ & c $\mathrm{F}$ & c F & c $F$ \\
\hline d $T$ & d $T$ & d F & d F & d F \\
\hline e $F$ & e $F$ & e $T$ & e F & e F \\
\hline
\end{tabular}

